\title{
Assessment of adrenoleukodystrophy lesions by high field MRS in non-sedated pediatric patients
}

\author{
G. Öz, PhD; I. Tkáč, PhD; L.R. Charnas, MD, PhD; I.Y. Choi, PhD; K.J. Bjoraker, PhD; E.G. Shapiro, PhD; \\ and R. Gruetter, $\mathrm{PhD}$
}

\begin{abstract}
Background: Early detection of white matter lesions in childhood-onset cerebral adrenoleukodystrophy (ALD) is important as hematopoietic cell transplantation (HCT), currently the only effective treatment, is beneficial only if performed early in the disease course. Objective: To establish reliable biochemical markers of cerebral disease progression in patients with ALD to aid in treatment planning. Methods: The authors used proton magnetic resonance spectroscopy (MRS) in combination with LCModel analysis to quantify brain metabolites in small volumes (3 to $16 \mathrm{~mL}$ ) in the occipital and frontal white matter and the splenium of the corpus callosum of 17 unsedated patients and 26 healthy volunteers (adult $\mathrm{n}=21$, age-matched $\mathrm{n}=5$ ) at 4 tesla. Results: Absolute concentrations of 12 metabolites were reliably determined, seven of which were established as markers of lesion development. Among these, creatine and choline containing compounds were the weakest markers while $N$-acetylaspartate, glutamine, and lipids + lactate were the strongest. The large extent of changes in the markers enabled detection of early neurochemical changes in lesion formation prior to detection of abnormalities by conventional MRI. Concentrations of a number of metabolites were also significantly different between normal appearing white matter of patients and controls indicating biochemical alterations in the absence of cerebral disease. Neurochemical improvements following HCT were measured in six patients. Conclusions: The progression of adrenoleukodystrophy, as well as effectiveness of its treatment, can be assessed with high precision using high field ${ }^{1} \mathrm{H}$ magnetic resonance spectroscopy in individual patients without the need for sedation.
\end{abstract}

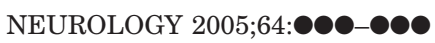

Fnu

X-linked adrenoleukodystrophy (ALD) is a disorder of $\beta$-oxidation of very long chain fatty acids (VLCFA) due to a defect in the ALDP (ALD protein) gene product in the peroxisomal membrane. ${ }^{1}$ Saturated VLCFA, particularly hexacosanoic (C26:0) and tetracosanoic (C24:0) acids, accumulate in many organs, particularly the adrenal gland and the brain. ${ }^{2}$ The minimum prevalence of the biochemical abnormality is 1 in 21,000 males. ${ }^{3}$ Several phenotypes exist, with the most severe being childhood-onset cerebral form (C-ALD), a progressive inflammatory demyelination of the cerebral white matter (WM). Approximately $35 \%$ of the boys with elevated VLCFA (biochemical ALD) develop C-ALD between 3 and 10 years of age. ${ }^{1}$ Once demyelination begins, progression of behavioral, cognitive, and neurologic deficits leads to vegetative state or death within a few years without effective treatment. The remaining boys will likely develop adrenomyeloneuropathy (AMN), with slowly progressive gait spasticity and sensory ataxia. This phenotype presents with a mean age at onset of 28 years. The pathology of AMN is different than C-ALD and is primarily a noninflammatory axonal degeneration of upper motor neurons and large fiber sensory pathways within spinal cord and peripheral nerves. ${ }^{1}$ Forty percent of men with AMN also have or develop cerebral involvement with inflammatory demyelination, similar to that seen in childhood. ${ }^{1}$

Biochemical ALD is easily diagnosed by elevated fasting plasma VLCFA levels. ${ }^{4}$ However, there are no reliable methods for predicting in whom C-ALD will develop. Currently the only therapy for C-ALD, hematopoietic cell transplantation (HCT; encompassing both bone marrow and cord blood transplant), is effective only if performed early in the course of cerebral disease. ${ }^{5}$ The effect of HCT on AMN is unknown. In addition, the morbidity and mortality of $\mathrm{HCT}$ are too great to use in asymptomatic boys with-

\section{$\overline{\text { See also page } \mathrm{XXX}}$}

From the Center for MR Research (Drs. Öz, Tkáč, Choi, and Gruetter), Department of Radiology, and Departments of Pediatrics (Drs. Charnas, Bjoraker, and Shapiro) and Neuroscience (Dr. Gruetter), University of Minnesota, Minneapolis. I.Y.C. is currently affiliated with The Nathan Kline Institute, Medical Physics, Orangeburg, NY.

Supported by a grant from the March of Dimes Birth Defects Foundation (E.G.S.). The Center for MR Research is supported by MIND Institute and a National Center for Research Resources (NCRR) biotechnology research resource grant P41RR08079 and the General Clinical Research Center at the University of Minnesota by NCRR grant M01RR00400.

Received June 21, 2004. Accepted in final form September 15, 2004.

Address correspondence and reprint requests to Dr. Gülin Öz or Rolf Gruetter, Center for MR Research, 2021 6th St. SE, Minneapolis, MN 55455; e-mail: gulin@cmrr.umn.edu 
out C-ALD. Similarly, HCT for boys with advanced disease (performance IQ less than 80 in previously normal boys) may exacerbate the disease progression and lead to poor functional outcome and death. ${ }^{6}$

C-ALD is currently diagnosed by detection of hyperintense regions in T2-weighted MRI before neurologic signs and symptoms. ${ }^{7-9}$ Gadolinium enhancement in ALD WM lesions predicts disease progression. ${ }^{10}$ Magnetization transfer MRI divides WM lesions into different zones, but adds no additional predictive value. ${ }^{11} \mathrm{~A}$ technique identifying the transition between biochemical ALD and C-ALD before structural damage is detected by imaging methods has the potential to improve medical care.

Biochemical changes such as altered energy and membrane metabolism and intercellular metabolite trafficking (glutamate-glutamine shuttling) are likely to be present before structural changes. Several groups using MR spectroscopy (MRS) found biochemical changes in the absence of abnormalities on T2-weighted MRI.8,12-15 These changes were limited to a few metabolites and their ratios (NAA, creatine, choline) at 1.5 tesla. Changes with disease progression in a larger number of metabolites were observed in pooled data sets at 2 tesla. ${ }^{9}$ We hypothesized that changes in more metabolites than previously studied would be quantified reliably with the higher sensitivity at 4 tesla. Furthermore, the measurement of these changes in single patients would represent an advance for disease monitoring.

We sought to establish neurochemical profiles of lesions of patients with ALD in comparison to their normal appearing WM (NAWM) and the WM of healthy controls and to determine the potential of high field MRS to detect early changes in this profile before abnormalities in T2-weighted MRI.

Methods. Patients and control subjects. Seventeen boys, 4 to 13 years, with the biochemical defect for ALD were studied after obtaining informed consent from their parents using procedures approved by the Institutional Review Board: Human Subjects Committee of the University of Minnesota. The mean age at the first MRS examination was $7.2 \pm 1.5$ years (mean $\pm \mathrm{SD}$, range 4 to 9 years). Twelve of these boys were studied longitudinally approximately every 6 months with an average duration of follow-up of $2.1 \pm 0.9$ years (range 0.6 to 3.4 years). The remaining five boys were studied once. Eight boys had normal MRI (T2-weighted and FLAIR) with a MRI Severity Score of $0 .{ }^{16}$ Nine boys had WM lesions visible as hyperintense regions in T2-weighted MRI. Six of them received HCT and were scanned before and after the transplantation.

Neurologic and neuropsychological examinations were performed at each visit. No neurologic abnormalities related to ALD were observed at baseline for any of the boys. Two boys had pre-existing neurologic abnormalities unrelated to ALD. Neuropsychological abnormalities related to ALD were seen at baseline in boys with hyperintense regions in T2-weighted MRI; however, none of them was considered to have disease that was too advanced for HCT.

Subjects were given the option to watch movies using an audio/ visual system ${ }^{17}$ during data collection. No sedation was used. During the entire study, a nurse inside the magnet room was monitoring the physiology of the subject by an attached pulse oximeter (In vivo Research Inc., Orlando, FL).

Spectra were acquired from 21 healthy adults (age $26.5 \pm 8.7$, range 18 to 47 years) and 5 age-matched controls (age $10.4 \pm 2.6$, range 6 to 12 years).
MRS. All studies were performed on a 4 tesla, $90 \mathrm{~cm}$ bore magnet (Oxford Magnet Technology, Oxford, UK) with an INOVA console (Varian, Palo Alto, CA) and a standard body gradient coil (Sonata, Siemens, Erlangen, Germany). A TEM volume coil ${ }^{18}$ was used as the NMR transceiver. Following positioning of the subject in the magnet, transverse multislice images were obtained with a RARE sequence (repetition time [TR] $=4$ seconds, echo train length $=8$, echo time $[\mathrm{TE}]=60 \mathrm{msec}, 7$ slices, 2 averages) for the selection of the volume of interest (VOI). Localization of ${ }^{1} \mathrm{H} M R$ spectra was achieved using ultra-short echo STEAM spectroscopy with $\mathrm{TE}=5 \mathrm{msec}$, mixing time $\mathrm{TM}=42 \mathrm{msec}$, and $\mathrm{TR}=4.5$ seconds. Water suppression was achieved using eight variable power RF pulses with optimized relaxation delays (VAPOR) which were interleaved with outer volume suppression to improve localization performance. ${ }^{19}$ All first- and second-order shims were adjusted using FASTMAP with echoplanar readout. ${ }^{20}$ For longitudinal studies voxel positions were chosen consistently to assess time dependent changes in the particular areas. Spectra were typically acquired from three brain regions: occipital and frontal WM (8 to $16 \mathrm{~mL}$ volumes, 5 to 6 minutes data acquisition) and the splenium of the corpus callosum ( 3 to $10 \mathrm{~mL}$ volumes, 5 to 10 minutes data acquisition). A typical session in the magnet did not exceed 50 minutes.

Spectra were acquired and saved as single scans, which were individually frequency and phase corrected to minimize the effects of motion on spectral linewidths and signal-to-noise ratio $(\mathrm{S} / \mathrm{N})$. Scans that showed evidence for substantial motion were excluded and the remaining scans summed. When insufficient $\mathrm{S} / \mathrm{N}$ did not permit correction of single acquisitions (such as in small voxels in the corpus callosum), data were averaged over four to eight scans and then corrected.

Spectral quantitation. The contribution of individual metabolites to the in vivo spectrum was quantified using LCModel. ${ }^{21}$ In this fully automatic method, the in vivo spectrum is modeled as a linear combination of adequately line-broadened individual metabolite spectra. The metabolites that are known to contribute to brain spectra are provided in a basis set, ${ }^{22}$ which in the current study included the following 20 compounds: Alanine (Ala), aspartate (Asp), glycerophosphoryl-choline (GPC), phosphoryl-choline (PCho), creatine (Cr), phosphocreatine (PCr), $\gamma$-aminobutyric acid (GABA), glucose (Glc), glutamine (Gln), glutamate (Glu), glutathione (GSH), myo-inositol (myo-Ins), lactate (Lac), $N$-acetylaspartate (NAA), $N$-acetyl-aspartyl-glutamate (NAAG), phosphorylethanolamine (PE), scyllo-inositol (scyllo-Ins), taurine (Tau), lipids, and macromolecules. The model metabolite spectra were obtained by simulation using previously reported chemical shifts and coupling constants. ${ }^{23}$ The macromolecule pattern was obtained by averaging inversion recovery spectra from the WM of five healthy subjects, where the STEAM parameters were optimized to null the metabolite resonances $(\mathrm{TR}=2$ seconds, inversion time $=0.675$ seconds). The pattern of the lipid model spectrum was established as follows: first, the two primary fatty acid resonances at 0.9 (methyl) and $1.3 \mathrm{ppm}$ (methylene, $\left.\left(\mathrm{CH}_{2}\right)_{\mathrm{n}}\right)$ were quantified in three in vivo spectra where the lipid contribution was strongest. The signal intensity ratio of the methyl and methylene peaks was very similar between these spectra; therefore the methyl-tomethylene ratio in the final lipid model spectrum was set to the average value ( 0.57$)$ obtained from these three spectra. Intensities of the smaller peaks at $1.60,2.05$, and $2.24 \mathrm{ppm}$ were based on literature $^{24}$ and fatty acid structure. Attempts to fit a peak at 2.8 ppm, where polyunsaturated fatty acids display a signal, indicated no contribution to the spectra at this chemical shift. The lipid concentrations were based on the methyl peak at $0.9 \mathrm{ppm}$ assuming one methyl group per fatty acyl moiety.

The intensity of the in vivo spectrum was calibrated using the water signal from the same voxel as an internal reference, ${ }^{25}$ assuming a water content of $72 \%$ for WM. ${ }^{26}$

Assessment of reliability in metabolite concentrations and statistical analysis. The criteria to select the reliable metabolite concentrations were based on the Cramér-Rao lower bounds (CRLB), which are estimates of the SD of the fit for each metabolite and are also determined by LCModel. Only results with a $\mathrm{CRLB} \leq 50 \%$ were included in the analysis. Concentrations with CRLB $>50 \%$ were classified as not detected. Only metabolites that had a CRLB below $50 \%$ in more than $70 \%$ of the spectra were included in the neurochemical profile. If the covariance between two metabolites was consistently high (correlation coefficient 
Table Average concentrations and Cramér-Rao lower bounds (CRLB) of cerebral metabolites in the occipital white matter

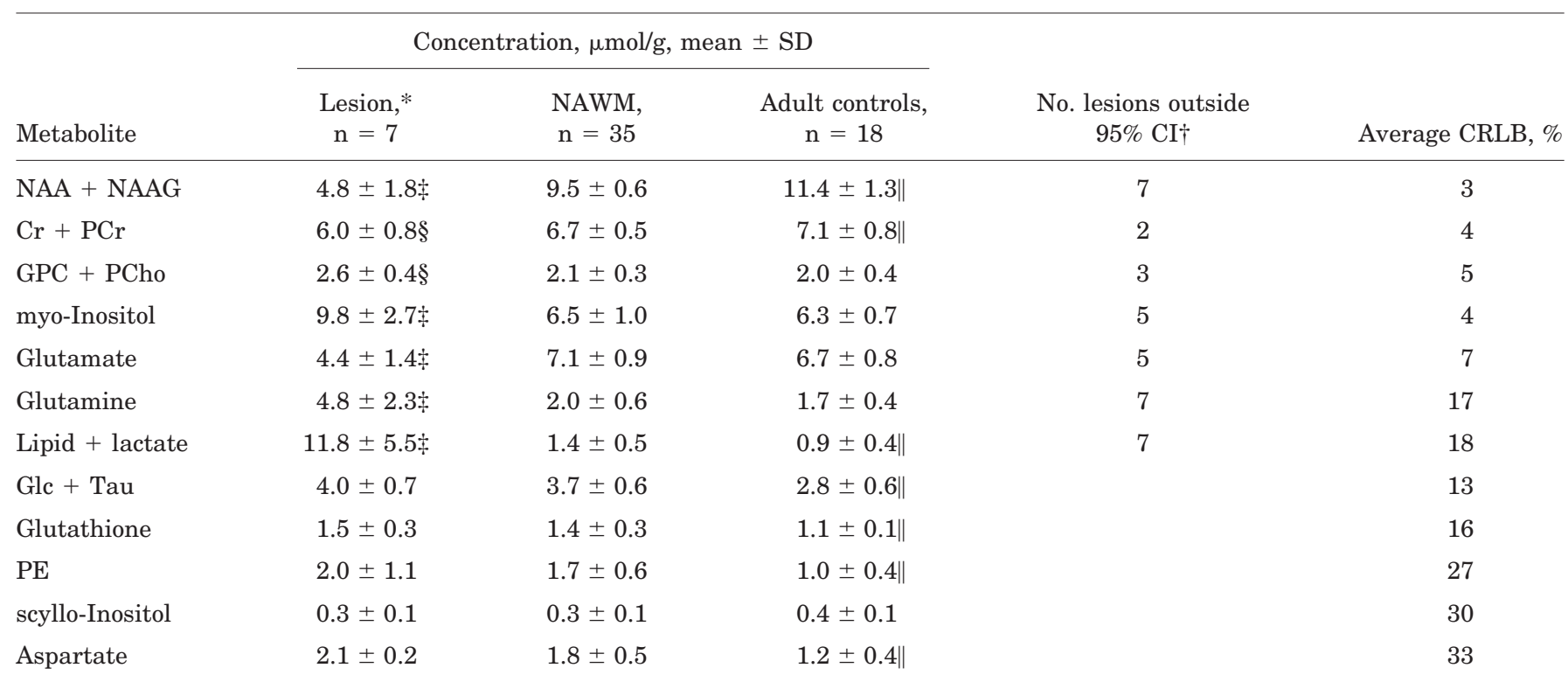

\footnotetext{
* Data acquired from lesions before hematopoietic cell transplantation.

$\dagger$ Number of lesions that were more than 2 SD different from the adult control values; i.e., outside the $95 \%$ CI.

$\ddagger p<0.0001$ Lesion vs both NAWM and control.

$\S p<0.01$ Lesion vs both NAWM and control.

$\| p<0.03$ Control vs both NAWM and lesion.
}

$\mathrm{NAWM}=$ normal-appearing white matter $\mathrm{NAA}=N$-acetylaspartate NAAG $=N$-acetyl-aspartyl-glutamate $; \mathrm{Cr}=\mathrm{creatine} ; \mathrm{PCr}=$ phosphocreatine; GPC = glycerophosphoryl-choline; Glc = glucose; Tau = taurine; PE = phosphorylethanolamine.

$<-0.5)$, such as in the case of $\mathrm{Cr}$ and $\mathrm{PCr}$, we chose to report their sum (table 1) even though their individual values were estimated by LCModel.

The concentrations of metabolites in healthy and lesioned tissue were compared using the two-tailed, unpaired Student's $t$ test.

Results. Constituents of the neurochemical profile of nonsedated patients and controls. When studying nonsedated children, motion of the subject is a frequent problem that reduces reliability of metabolite quantification. We found that spectral intensity losses can be as high as 30 to $40 \%$ due to scan-to-scan phase and frequency changes. Phase and frequency correction of individual scans eliminated these losses. The correction scheme was verified by acquiring spectra from the same brain region in the presence and absence of motion and identical concentrations were obtained from these spectra upon applying the single scan correction.

Absolute metabolite concentrations in three known sites of ALD lesions, namely occipital and frontal WM and corpus callosum, were determined by deconvolution of the in vivo ${ }^{1} \mathrm{H}$ MR spectra into model spectra of 20 metabolites. In addition to compounds previously described to contribute to spectra, ${ }^{22}$ the contribution of lipid signals was assessed because spectra from lesions displayed fatty acid peaks at 0.9 and $1.3 \mathrm{ppm}$, in some cases comparable in intensity to creatine. The inclusion of lipids in the basis set resulted in significant improvement of the LCModel fit (as judged by CRLB, residuals and baseline) in all lesions. Conversely, omission of the lipid model spectrum from the basis set resulted in overestimation of lactate and macromolecule contributions in lesions.

The reliability of obtained concentrations was judged based on Cramér-Rao lower bounds (see Methods for crite- ria). Of the 20 compounds analyzed, Ala and GABA were excluded and scyllo-Ins, Asp, and PE were considered borderline reliable. Substantial covariance of concentrations was found in five cases, so that NAA + NAAG, $\mathrm{Cr}+\mathrm{PCr}$, $\mathrm{GPC}+$ PCho (from here on referred to as NAA, Cr, and Cho), Lipid + Lac, and Glc + Tau were included in the neurochemical profile instead of the concentrations of individual compounds. Therefore 12 statistically independent concentrations were evaluated (see table 1). The metabolite concentrations in the frontal WM and corpus callosum were similar to those in the occipital WM. Similarly, the average CRLB values in frontal WM were comparable to occipital WM. The CRLB in the corpus callosum were somewhat higher due to the lower $\mathrm{S} / \mathrm{N}$ in the smaller VOIs.

Neurochemical alterations in cerebral and non-cerebral (biochemical) ALD. Proton MR spectra acquired from WM lesions of patients with C-ALD displayed extensive changes when compared to those from the NAWM of patients with ALD (figure 1), as well as control subjects. NAA, Cr, and Glu were significantly lower and Cho, myoIns, Gln, and Lipid + Lac were higher in lesions than both NAWM of patients and healthy controls (see table 1 ). The concentrations of three of these lesion markers (NAA, Gln, Lipid + Lac) were more than two SDs different than controls in all lesions in occipital WM, demonstrating the sensitivity of the MRS method to identify cerebral disease.

The ratio of Glu-to-Gln was compared to the Cho-to-Cr ratio, which has been extensively used as a disease marker. ${ }^{12,14}$ Cho-to-Cr was only $50 \%$ higher in lesions in the frontal and occipital WM while Glu-to-Gln was three- to fourfold lower (figure 2). The difference in the Cho-to-Cr ratio between lesion and control values in the splenium of the corpus callosum did not reach statistical significance. 


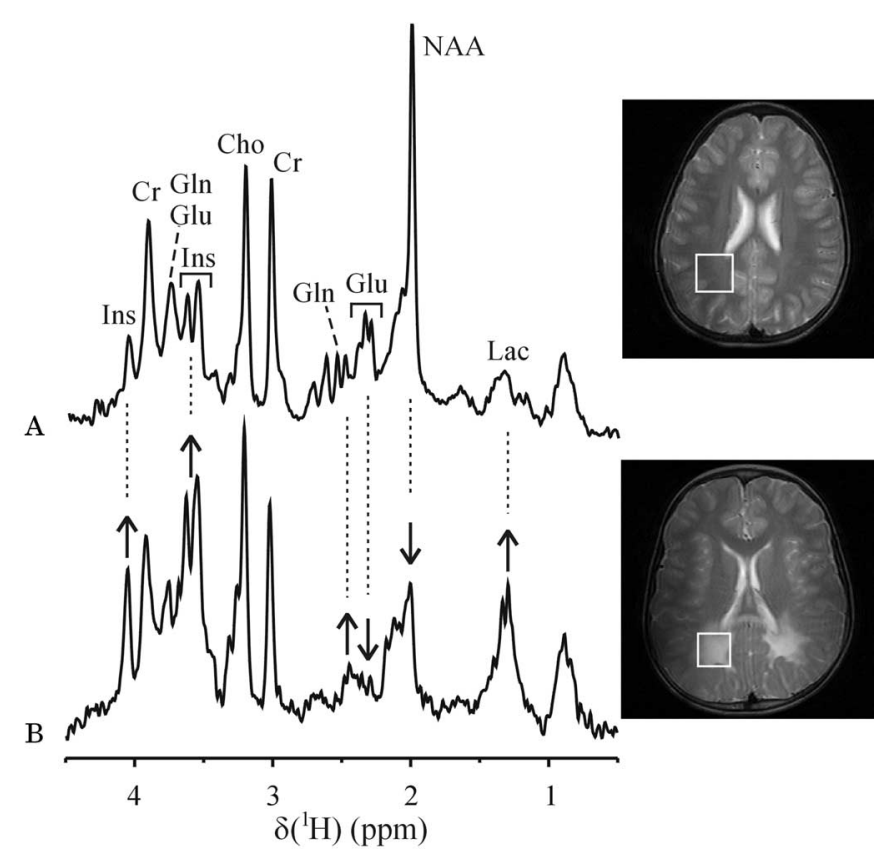

Figure 1. Proton MR spectrum from the normalappearing white matter (NAWM) of a 6-year-old asymptomatic patient with adrenoleukodystrophy (ALD) (A) in comparison to the spectrum from a lesion of a 9-year-old patient with cerebral $A L D(B)$. The voxels in the occipital WM are shown on the T2-weighted images, VOI in A was $16 \mathrm{~mL}$ and in $B 8 \mathrm{~mL}$. The major metabolite peaks are labeled (Ins: myo-inositol, $\mathrm{Cr}: \mathrm{Cr}+\mathrm{PCr}$, total creatine, Gln: glutamine, Glu: glutamate, Cho: PCho $+G P C$, choline containing compounds, NAA: NAA+NAAG, total $\mathrm{N}$-acetylaspartate, Lac: lactate). Changes in the lesion are shown with arrows.

Conversely, the Glu-to-Gln ratio in the corpus callosum lesions was $\sim$ threefold lower than controls $(p<0.003)$, as in the frontal and occipital WM lesions.

The distribution of metabolite concentrations among subject groups was further assessed by analyzing correlation plots of metabolite concentrations, such as Gln vs Glu and Lipid + Lac vs myo-Ins (figure 3). The distribution of metabolite concentrations in lesions was distinctly different from that in NAWM as well as in adult and agematched controls. One lesion data point in the Gln vs Glu plot (see figure 3B, circle) was close to those of controls, but the other constituents of the neurochemical profile in this voxel clearly indicated a lesion pattern.

Neurochemical differences were also detected between NAWM of patients and controls (see table 1). For example, no lipid contribution was measured in control brains, while NAWM displayed significantly increased Lipid + Lac values. To determine if the differences observed were due to the age difference between the patient and the control group, data were acquired from five age-matched healthy subjects. GSH, PE, and Glc + Tau were indeed different between age-matched and adult controls $(p<0.05)$.

Neurochemical changes in the transition from noncerebral to cerebral $A L D$. The two outlier data points in the Lipid + Lac vs myo-Ins plot (figure 3A, circle) display neurochemical changes due to lesion development that were detected before appearance of abnormalities in T2weighted MRI. These data were acquired from NAWM of
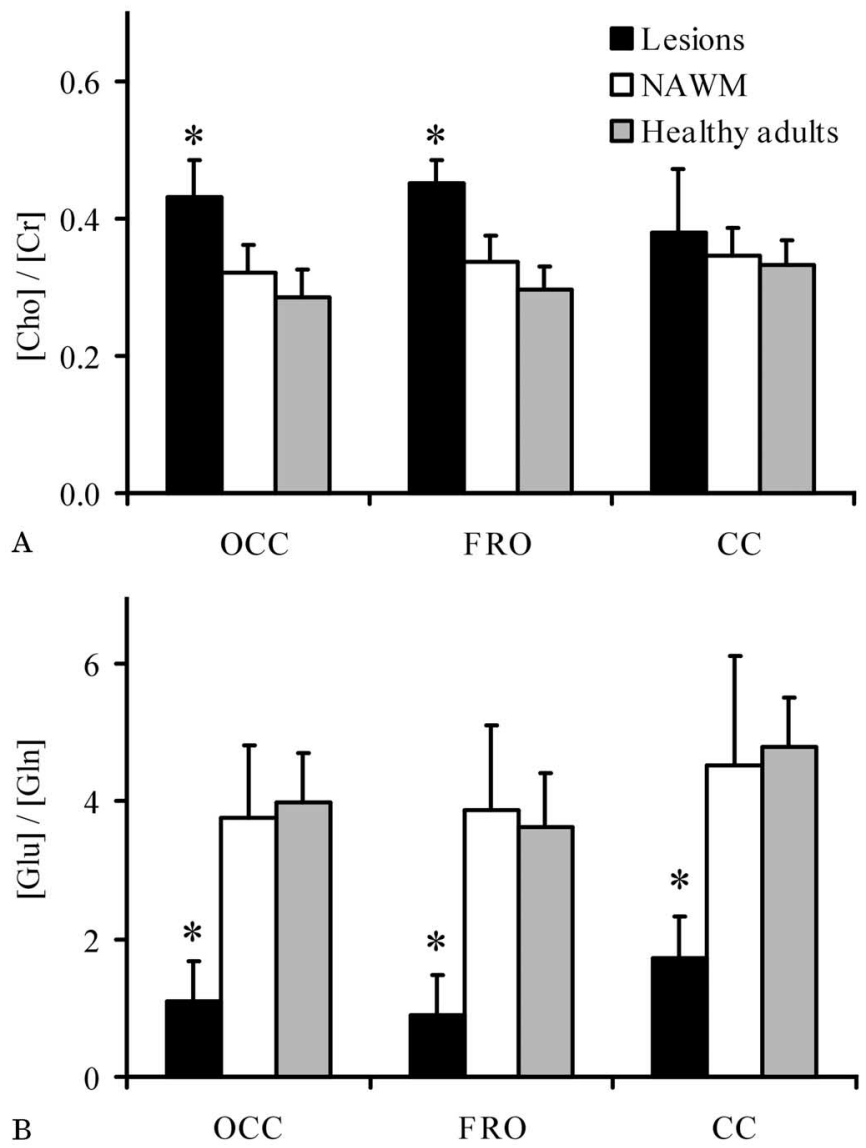

Figure 2. Comparison of choline-to-creatine (A) and glutamate-to-glutamine (B) concentration ratios in different brain regions of patients (lesions, normal-appearing white matter [NAWM]) and healthy adults. Cho refers to $\mathrm{GPC}+\mathrm{PCho}, \mathrm{Cr}$ to $\mathrm{PCr}+\mathrm{Cr}$, OCC to occipital WM, FRO to frontal WM, and CC to splenium of corpus callosum. Error bars indicate SDs. *p $<0.003$ for lesion vs both NAWM and healthy adult values.

patients who had lesions in other areas of the brain. In both cases lesion progression into the VOI of the MRS measurement became apparent only at subsequent MRI examinations.

Another example of early detection of lesion development is shown in figure 4 . The frontal WM spectrum shown was acquired from a region with borderline hyperintensity in the T2-weighted image, however, quantification of this spectrum revealed a full lesion profile (low NAA, Glu, high Cho, myo-Ins, Gln). This profile was consistent with worsened anterior neuropsychological scores (executive function, speed, motor, memory/learning).

Neurochemical changes following HCT. Six patients with C-ALD were examined before and up to 2.3 years after HCT. In addition to reduced Cho and stabilized or somewhat reduced NAA values (with the exception of one patient), these patients displayed normalized/reduced Gln and Lipid + Lac concentrations (figure 5). Their Glu remained low or decreased further and myo-Ins remained high or increased further. The spectra of one of these patients after the HCT are shown in figure 4. While the occipital WM spectrum was still very different from healthy WM with the high myo-Ins and low NAA peaks, 

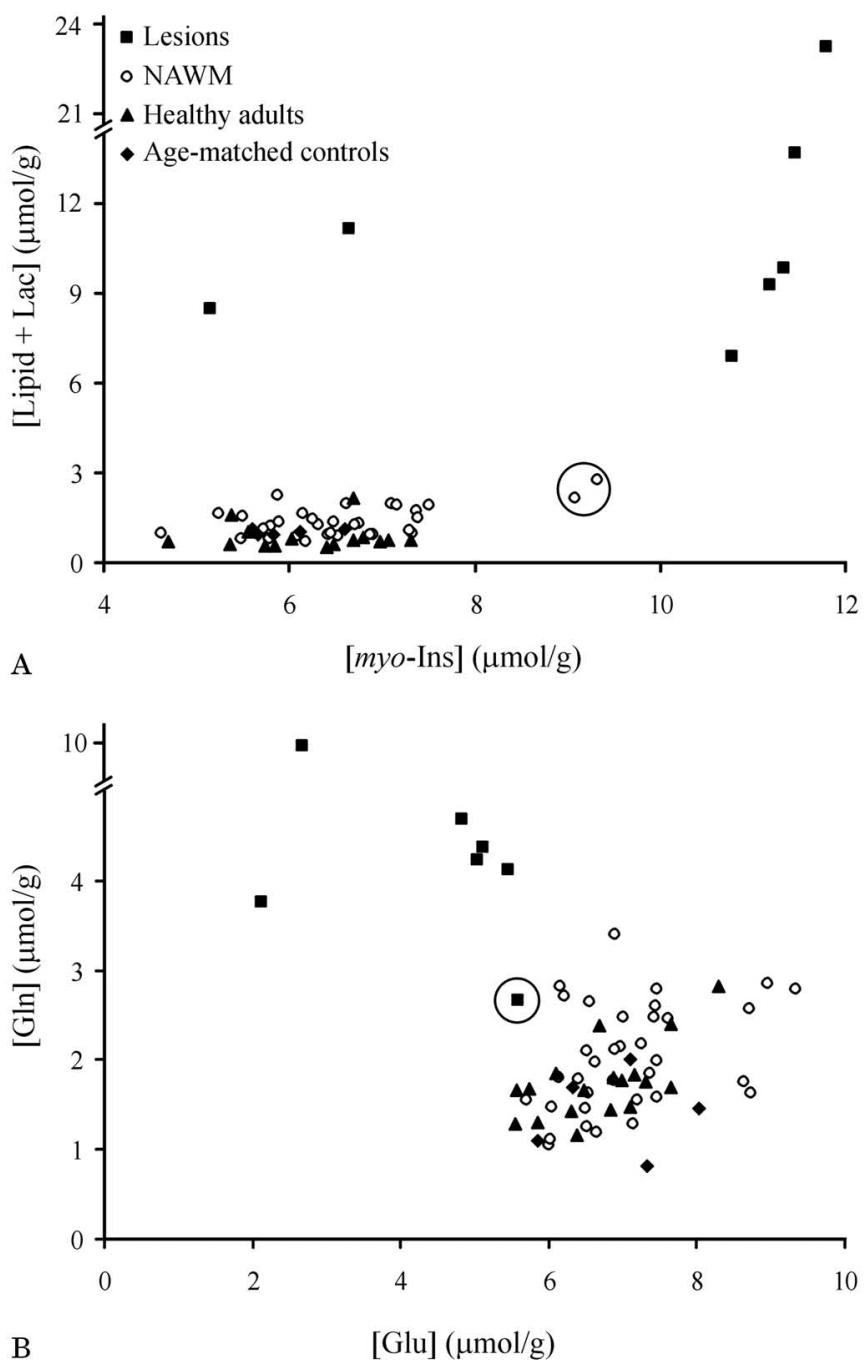

Figure 3. Correlation plots of metabolite concentrations in occipital white matter in different groups of subjects. (A) Lipid + lactate vs myo-inositol; B) glutamine vs glutamate. The large dynamic range of concentrations from healthy to lesioned tissue is illustrated. Lesion data were acquired before hematopoietic cell transplantation. The outliers in A shown with a circle around them display early neurochemical changes in lesion formation. The outlier in B (circle) was acquired from a lesion that displayed the characteristic changes in the other markers (high Cho, myo-Ins, Lipid + Lac, low NAA). NAWM = normalappearing white matter.

the improvement in the neurochemical profile was evident from the normalized Cho-to-Cr ratio. Spectral quantitation further revealed a reduction in Gln and Lipid + Lac. These improvements were consistent with an improved posterior neuropsychological score (visual and auditory perception).

Discussion. Quick, safe, precise, and reliable measurement of disease markers in individuals is needed to apply a method to patient monitoring. We describe the reliable measurement of a 12-component neurochemical profile with high precision in three WM regions involved in early C-ALD in less than 1 hour. We did not use sedation, which reduced both the risk

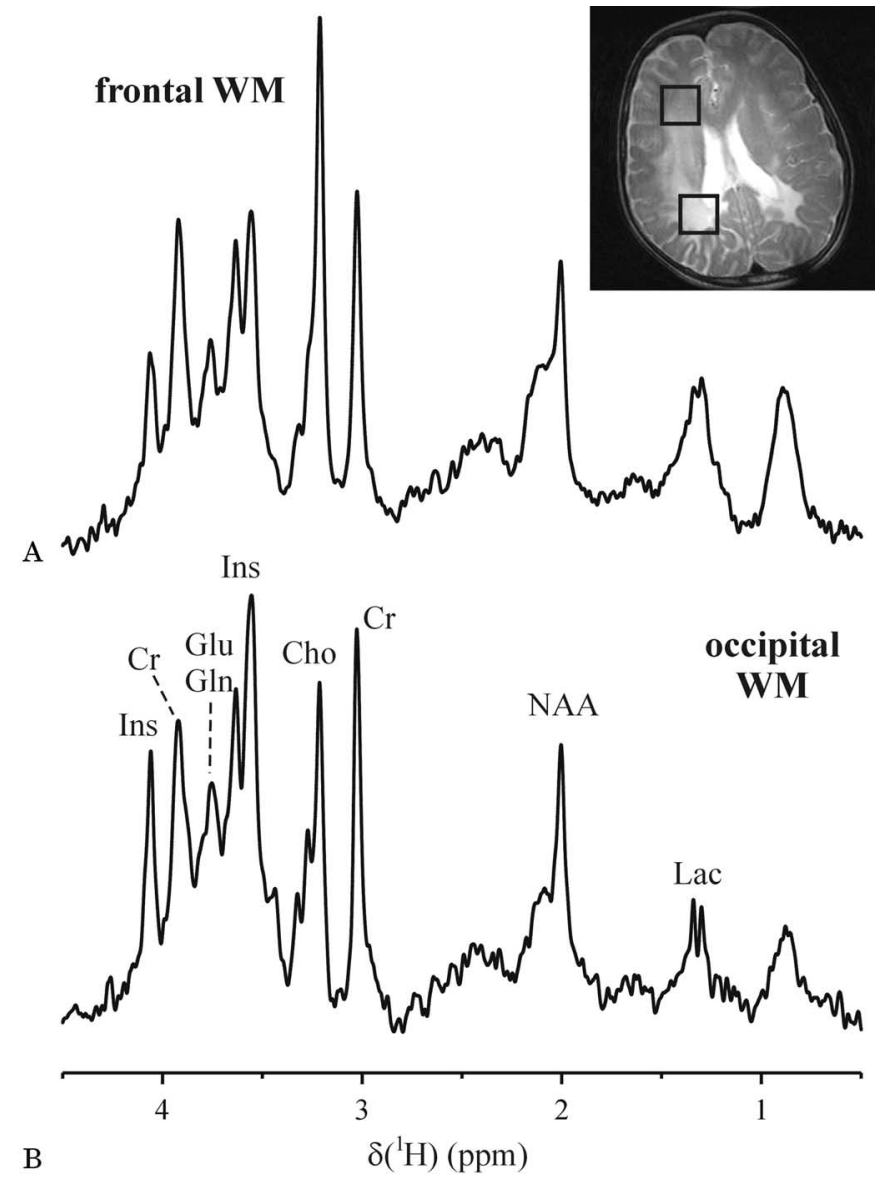

Figure 4. Proton MRS of a patient with cerebral adrenoleukodystrophy after hematopoietic cell transplantation. The voxels $(16 \mathrm{~mL})$ in the frontal and occipital white matter (WM) are shown on the T2-weighted image. The lesioned occipital WM (B) shows an improved neurochemical profile as a result of transplant (reduced Cho, Gln, Lipid + Lac), while the frontal WM (A) spectrum acquired from a region with borderline MRI hyperintensity displays a lesion pattern (reduced NAA, Glu, increased Cho, myoIns, Gln).

and the time and effort involved in each scan. This is the first study where such an extensive neurochemical profile was assessed noninvasively in individual patients to follow a neurologic condition.

Typically MRS studies of the brain evaluate three to five metabolites (NAA, Cr, Cho, myo-Ins, Lac) or their ratios, while we assessed concentrations of 12 metabolites, 7 of which were metabolic markers of ALD lesions (see table 1). Measurement of absolute concentrations avoids ambiguities associated with relative measurements and although it is more prone to averaging errors caused by motion, which occur frequently in non-sedated children, we minimized these potential errors by acquiring the spectra in single scans. This approach substantially improved the spectral quality (resolution and $\mathrm{S} / \mathrm{N}$ ) and the reliability of metabolite quantification and should be applicable to 3 tesla magnets that are increasingly being used in the clinical setting. At lower magnetic fields, which preclude such an approach, statistically 


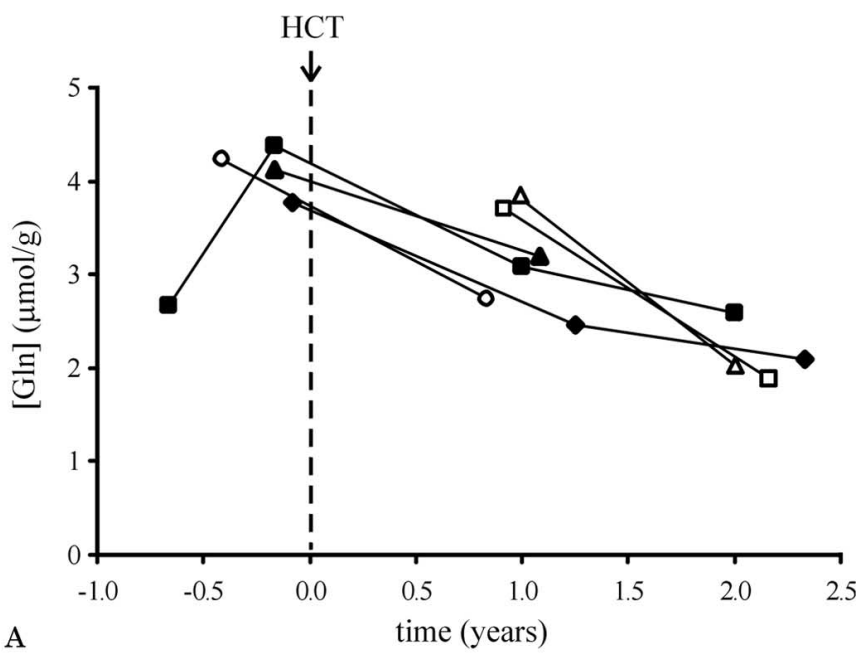

A

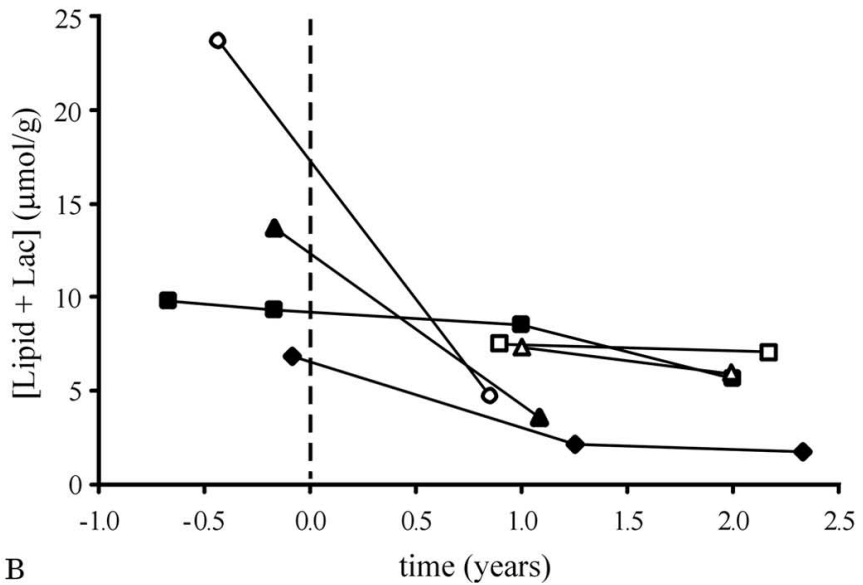

Figure 5. Decrease of glutamine (A) and lipids + lactate (B) in occipital white matter lesions over time after hematopoietic cell transplantation (HCT). The different symbols represent different patients. HCT was performed at $t=0$. All patients were scanned before HCT, however in two cases $(\square, \triangle)$ spectra were not acquired in the same locations as after HCT, therefore only data after HCT are shown.

significant differences between subject groups may be obscured. ${ }^{8}$ The high precision in our concentration measurements was evident from the low CRLB (see table 1). Changes greater than twice the CRLB can be detected in individual spectra with 95\% confidence. ${ }^{27}$ For example, changes in Glu concentrations of $14 \%$ are detectable with our protocol.

Abnormalities were identified using metabolite correlation plots (see figure 3). There is tight distribution of healthy control and NAWM values in the Lipid + Lac vs myo-Ins plot. The lesion values are more variable, likely because they represent different disease stages. This suggests that lesion progression can be followed in individual patients with C-ALD over time with our method. Indeed, we detected neurochemical improvements in lesions of each of the six patients after HCT (see figure 5). Furthermore, we detected a starting lesion in the NAWM of two patients (see figure 3A, circle) and a full blown lesion in a region with borderline hyperin- tensity in the T2-weighted image (see figure 4). Such early detection of changes in the numerous lesion markers is likely to increase the predictive value of the MRS method. In contrast, a low NAA-to-Cho ratio was identified as the sole predictor of C-ALD progression in a retrospective MRS imaging (MRSI) study. ${ }^{28}$ Although the coverage of whole brain slices by the MRSI technique may be advantageous in diseases where the locations of lesion development are more uncertain, such as multiple sclerosis, the extensive profile provided by single voxel spectroscopy outweighs this advantage in a disease like ALD where all sites of possible cerebral involvement can be covered in a reasonably short scan time. Another major advantage of single voxel spectroscopy over MRSI is that the effects of motion can be minimized.

The current study extended previous reports of low NAA, high Cho, and myo-Ins, ${ }^{8,9,13}$ as well as occasional observations of high $\mathrm{Lac}^{8,9,12-14}$ and lipids ${ }^{12,13,29}$ in ALD lesions. Surprisingly, Cho and Cr, two compounds extensively used in assessing abnormalities, were the weakest markers (see figure 2 ; also see $p$ values and number of lesions that were more than 2 SD different from adult controls in table 1). Despite the evaluation of the sum of Lipid and Lac, due to the overlap of their signals at $\sim 1.3 \mathrm{ppm}$, this measurement was one of the best markers of advancing disease (see figure 3A). The Lipid + Lac increase was probably due to both lactate and mobile lipids because we detected a lactate doublet with the expected $7 \mathrm{~Hz}$ homonuclear coupling in some patients (see figures 1 and 3). A reduction in $\mathrm{Cr}$ was observed in one other study in advanced lesions. ${ }^{9}$ Similarly, the decrease in Glu and increase in Gln were observed in one previous study, but only when using group averages. ${ }^{9}$ In the present study Glu and Gln were reliably measured in individual patients with ALD for the first time and their ratio was highly sensitive to the presence of lesions (see figure 2).

These results indicate that several major features of ALD pathology can be assessed in individual patients using MRS at 4 tesla, including neuroaxonal loss, gliosis, demyelination, and macrophage infiltration. ${ }^{1}$ Reduced NAA and Glu levels in lesions are likely a result of neuroaxonal loss while increased Gln and myo-Ins are possibly associated with gliosis. ${ }^{9,30}$ Cho is thought to increase during active demyelination, ${ }^{9}$ whereas increases in lactate have been ascribed to the anaerobic metabolism of infiltrating macrophages..$^{8,9,12-14}$ Reduced $\mathrm{Cr}$ may indicate disturbances in energy metabolism.

Of special interest was the assessment of a lipid contribution to the lesion spectra since ALD is a disorder of lipid metabolism. Incomplete elimination of intense subcutaneous fat signals may confound accurate measurement of mobile lipids in the brain. ${ }^{8}$ In our data, the signals of the mobile lipids were in-phase relative to the rest of the spectrum and the lipid contribution was consistently observed in all lesions but never in healthy tissue, indicating that the lipid resonances originated from the VOI. Fur- 
thermore, the localization method used herein provides a high degree of subcutaneous lipid signal suppression. An intriguing question is whether the detected mobile lipid resonances stem from the saturated VLCFA that accumulate in ALD, as suggested earlier. ${ }^{13}$ We tentatively ascribed the lipid contribution to a mixture of saturated and monounsaturated fatty acids since the spectra displayed a small peak at $5.3 \mathrm{ppm}$ indicative of double bonds, but none at $2.8 \mathrm{ppm}$ indicating lack of polyunsaturated fatty acids. ${ }^{31}$ Furthermore, the methyl-to-methylene ratio corresponded to short fatty acyl chains suggesting that the lipid resonances appear due to increased turnover of myelin lipids, rather than VLCFA accumulation. However, binding of VLCFA to large structures in the cell, such as the proteolipid protein, may also result in the observed methyl-to-methylene ratio. Furthermore, the evidence for unsaturation in our data does not rule out the possibility that the detected lipids represent VLCFA since monounsaturated VLCFA increase in phospho- and proteolipids and gangliosides in the ALD brain along with saturated VLCFA. ${ }^{1}$

Biochemical alterations were present in the ALD brain even in the absence of cerebral disease (see table 1). Among these, decreased NAA was previously reported. ${ }^{8,15}$ A significant increase in Lipid + Lac and decrease in $\mathrm{Cr}$ were detected. The increase of Lipid + Lac may be an indication of increased VLCFA in intact brain regions as observed previously by histopathology. ${ }^{1}$ Other differences between NAWM of patients with ALD and adult controls (GSH, PE, and Glc + Tau) were possibly due to the difference in age. However, these metabolites are not strongly represented in the spectra and more subjects are necessary to determine whether these metabolites change developmentally in the age range (4 to 13) of this study. Although most metabolic changes in the developing human brain appear to occur in the first 2 to 3 years, ${ }^{32-34}$ to our knowledge no studies have evaluated the current neurochemical profile in the same age range.

We followed neurochemical changes in response to HCT, for which MRI may not be the ideal tool..$^{35}$ Indeed, in patients who had undergone HCT the hyperintense regions on T2-weighted images after transplant were either similar to or more extensive than those before HCT. Conversely, some metabolite concentrations that were abnormal prior to HCT approached control values after HCT and continued to improve over time (see figure 5). Our results after HCT agree with and extend previous observations in two studies that reported decreased Cho, conserved or decreased NAA, and conserved or increased myoIns. ${ }^{9,36}$ We measured three additional metabolites (Glu, Gln, Lipid + Lac) that changed after the HCT and the consistent reduction of two of these $(\mathrm{Gln}$, Lipid + Lac) suggested improvements in the neurochemical profile. Which of these markers is associated with a good outcome in the long term remains to be determined.
In previous studies the reduced Cho levels after HCT were interpreted as arrested demyelination and the high myo-Ins as a consequence of remaining high content of glial cells and further reactive gliosis. ${ }^{9,36}$ However, the normalized Gln concentrations, if taken to represent glial cell volume, suggest reversal of gliosis. Alternatively, the restored Gln concentrations could result from a metabolic adaptation by the brain. Myo-Ins, on the other hand, is involved in a number of different functions in the cell, from serving as a precursor for lipid synthesis to acting as an idiogenic osmolyte. ${ }^{37}$ Therefore, myo-Ins concentration may not exclusively reflect glial volume. The decrease of Lipid + Lac levels may be due to the mobile lipids having been metabolized by the donor microglia that entered the brain as a result of the HCT. ${ }^{38}$ Even though Lac decreases following HCT were conceivable, the characteristic doublet at 1.3 ppm was still visible in some patients (see figure 4), suggesting that lactate remained elevated since it is difficult to detect in normal brain tissue. Finally, the unchanged/reduced Glu and NAA concentrations after the HCT were consistent with an irreversible loss of neuronal tissue.

\section{Acknowledgment}

The authors thank the nurses of the General Clinical Research Center for their support of this study, the staff of the Center for MR Research for maintaining and supporting the NMR system, and Dr. Stephen Provencher for discussions regarding the addition of lipid model spectra to the LCModel basis set.

\section{References}

1. Moser HW, Smith KD, Watkins PA, Powers J, Moser AB. X-linked adrenoleukodystrophy. In: Scriver CR, ed. The metabolic and molecular bases of inherited disease. 8 ed. New York: McGraw-Hill, 2001;32573301 .

2. Moser HW, Loes DJ, Melhem ER, et al. X-linked adrenoleukodystrophy: overview and prognosis as a function of age and brain magnetic resonance imaging abnormality. A study involving 372 patients. Neuropediatrics 2000;31:227-239.

3. Bezman L, Moser AB, Raymond GV, et al. Adrenoleukodystrophy: incidence, new mutation rate, and results of extended family screening. Ann Neurol 2001;49:512-517.

4. Moser AB, Kreiter N, Bezman L, et al. Plasma very long chain fatty acids in 3,000 peroxisome disease patients and 29,000 controls. Ann Neurol 1999;45:100-110.

5. Shapiro E, Krivit W, Lockman L, et al. Long-term effect of bone-marrow transplantation for childhood-onset cerebral X-linked adrenoleukodystrophy. Lancet 2000;356:713-718.

6. Charnas L, Shapiro E, Krivit W, et al. Performance IQ (PIQ) and MRI Severity Score (MRI-SS) but not Verbal IQ (VIQ) predict survival and disability after hematopoietic stem cell transplantation (HSCT) in childhood onset cerebral adrenoleukodystrophy (CALD). Am J Hum Genet 2002;71(suppl):A580. Abstract.

7. Aubourg P, Sellier N, Chaussain JL, Kalifa G. MRI detects cerebral involvement in neurologically asymptomatic patients with adrenoleukodystrophy. Neurology 1989;39:1619-1621.

8. Kruse B, Barker PB, van Zijl PC, Duyn JH, Moonen CT, Moser HW. Multislice proton magnetic resonance spectroscopic imaging in X-linked adrenoleukodystrophy. Ann Neurol 1994;36:595-608.

9. Pouwels PJ, Kruse B, Korenke GC, Mao X, Hanefeld FA, Frahm J. Quantitative proton magnetic resonance spectroscopy of childhood adrenoleukodystrophy. Neuropediatrics 1998;29:254-264.

10. Melhem ER, Loes DJ, Georgiades CS, Raymond GV, Moser HW $\mathrm{X}$-linked adrenoleukodystrophy: the role of contrast-enhanced MR imaging in predicting disease progression. AJNR Am J Neuroradiol 2000; 21:839-844.

11. Melhem ER, Breiter SN, Ulug AM, Raymond GV, Moser HW. Improved tissue characterization in adrenoleukodystrophy using magnetization transfer imaging. AJR Am J Roentgenol 1996;166:689-695.

12. Tzika AA, Ball WS, Jr., Vigneron DB, Dunn RS, Nelson SJ, Kirks DR. Childhood adrenoleukodystrophy: assessment with proton MR spectroscopy. Radiology 1993;189:467-480. 
13. Confort-Gouny S, Vion-Dury J, Chabrol B, Nicoli F, Cozzone PJ. Localised proton magnetic resonance spectroscopy in X-linked adrenoleukodystrophy. Neuroradiology 1995;37:568-575.

14. Rajanayagam V, Grad J, Krivit W, et al. Proton MR spectroscopy of childhood adrenoleukodystrophy. AJNR Am J Neuroradiol 1996;17: 1013-1024.

15. Eichler FS, Itoh R, Barker PB, et al. Proton MR spectroscopic and diffusion tensor brain MR imaging in X-linked adrenoleukodystrophy: initial experience. Radiology 2002;225:245-252.

16. Loes DJ, Hite S, Moser H, et al. Adrenoleukodystrophy: a scoring method for brain MR observations. AJNR Am J Neuroradiol 1994;15: 1761-1766.

17. Harned RK, 2nd, Strain JD. MRI-compatible audio/visual system: impact on pediatric sedation. Pediatr Radiol 2001;31:247-250.

18. Vaughan JT, Hetherington HP, Otu JO, Pan JW, Pohost GM. High frequency volume coils for clinical NMR imaging and spectroscopy. Magn Reson Med 1994;32:206-218.

19. Tkáč I, Starcuk Z, Choi I-Y, Gruetter R. In vivo ${ }^{1} \mathrm{H}$ NMR spectroscopy of rat brain at $1 \mathrm{~ms}$ echo time. Magn Reson Med 1999;41:649-656.

20. Gruetter R, Tkáč I. Field mapping without reference scan using asymmetric echo-planar techniques. Magn Reson Med 2000;43:319-323.

21. Provencher SW. Estimation of metabolite concentrations from localized in vivo proton NMR spectra. Magn Reson Med 1993;30:672-679.

22. Pfeuffer J, Tkáč I, Provencher SW, Gruetter R. Toward an in vivo neurochemical profile: quantification of 18 metabolites in short-echotime ${ }^{1} \mathrm{H}$ NMR spectra of the rat brain. J Magn Reson 1999;141:104120 .

23. Govindaraju V, Young K, Maudsley AA. Proton NMR chemical shifts and coupling constants for brain metabolites. NMR Biomed 2000;13: 129-153.

24. Seeger U, Klose U, Mader I, Grodd W, Nagele T. Parameterized evaluation of macromolecules and lipids in proton MR spectroscopy of brain diseases. Magn Reson Med 2003;49:19-28.

25. Kreis R, Ernst T, Ross BD. Absolute quantitation of water and metabolites in the human brain. II. Metabolite concentrations. J Magn Reson 1993;102:9-19.

26. Siegel GJ, ed. Basic neurochemistry: molecular, cellular and medical aspects. 6 ed. Philadelphia: Lippincott-Raven Publishers, 1999.
27. Provencher SW. LCModel \& LCMgui User's Manual, 2001.

28. Eichler FS, Barker PB, Cox C, et al. Proton MR spectroscopic imaging predicts lesion progression on MRI in X-linked adrenoleukodystrophy. Neurology 2002;58:901-907.

29. Engelbrecht V, Rassek M, Gartner J, Kahn T, Modder U. The value of new MRI techniques in adrenoleukodystrophy. Pediatr Radiol 1997;27: 207-215.

30. Brand A, Richter-Landsberg C, Leibfritz D. Multinuclear NMR studies on the energy metabolism of glial and neuronal cells. Dev Neurosci 1993;15:289-298.

31. Evanochko WT, Pohost GM. Structural studies of NMR detected lipids in myocardial ischemia. NMR Biomed 1994;7:269-277.

32. van der Knaap MS, van der Grond J, van Rijen PC, Faber JA, Valk J, Willemse K. Age-dependent changes in localized proton and phosphorus MR spectroscopy of the brain. Radiology 1990;176:509-515.

33. Kreis R, Ernst T, Ross BD. Development of the human brain: in vivo quantification of metabolite and water content with proton magnetic resonance spectroscopy. Magn Reson Med 1993;30:424-437.

34. Pouwels PJ, Brockmann K, Kruse B, et al. Regional age dependence of human brain metabolites from infancy to adulthood as detected by quantitative localized proton MRS. Pediatr Res 1999;46:474-485.

35. Loes DJ, Stillman AE, Hite S, et al. Childhood cerebral form of adrenoleukodystrophy: short-term effect of bone marrow transplantation on brain MR observations. AJNR Am J Neuroradiol 1994;15:1767-1771.

36. Wilken B, Dechent P, Brockmann K, et al. Quantitative proton magnetic resonance spectroscopy of children with adrenoleukodystrophy before and after hematopoietic stem cell transplantation. Neuropediatrics $2003 ; 34: 237-246$.

37. Fisher SK, Novak JE, Agranoff BW. Inositol and higher inositol phosphates in neural tissues: homeostasis, metabolism and functional significance. J Neurochem 2002;82:736-754.

38. Krivit W, Peters C, Shapiro EG. Bone marrow transplantation as effective treatment of central nervous system disease in globoid cell leukodystrophy, metachromatic leukodystrophy, adrenoleukodystrophy, mannosidosis, fucosidosis, aspartylglucosaminuria, Hurler, MaroteauxLamy, and Sly syndromes, and Gaucher disease type III. Curr Opin Neurol 1999;12:167-176. 